\title{
Evidence-Based Optimization of the Mandarin Version of the Post Stroke Checklist: A Mixed Methods Study
}

\author{
Miao Jia \\ Nanfang Hospital \\ Huiqi Lu \\ Nanfang Hospital \\ Yan Li \\ Guangdong 999 Brain Hospital \\ Zhihong Liu \\ Guangdong 999 Brain Hospital \\ Jinyi Tu \\ Nanfang Hospital \\ Yue Liu \\ Nanfang Hospital \\ Simin Huang \\ Nanfang Hospital \\ Jinjun Li \\ Nanfang Hospital \\ Hongzhen Zhou ( $\nabla$ zhouhz_smu@126.com ) \\ Nanfang Hospital
}

\section{Research Article}

Keywords: Evidence-Based Practice, Stroke Rehabilitation, Long-Term Care, Tele-rehabilitation, Quality of Life

Posted Date: October 18th, 2021

DOI: https://doi.org/10.21203/rs.3.rs-838849/v1

License: (c) (1) This work is licensed under a Creative Commons Attribution 4.0 International License.

Read Full License 


\section{Abstract}

Background: The Post Stroke Checklist has been proved to be feasible and useful in standardizing the process for long-term post-stroke care and we developed a Mandarin version of it (M-PSC). However, it is not capable of enforcing follow-up care after stroke and further optimization is needed.

Methods: Participants were consecutively recruited from 13 departments in a specialized hospital on the discharge date. The trained clinicians contacted them by telephone calls at six months since the most recent diagnosis. A satisfaction questionnaire was sent to the participant whilst clinicians completed a satisfaction questionnaire and the Pragmatic Face and Content Validity Test (PRAC-Test). Then, a debriefing meeting was held to discuss potential problems of the M-PSC. Quantitative and qualitative data were both collected from April 2021 to May 2021.

Results: A total of 167 individuals consented to take part in the study.113 participants completed the assessment at six months and three were excluded due to missing data. The M-PSC identified a wide range of unmet needs related to stroke and at least one need was reported in $76.4 \%$ of participants. The average time taken to administer the M-PSC was 8 mins. Satisfaction ratings were high for participants $(8.5 / 10)$ and clinicians (9.6/10). Totally 31 further screenings were conducted and 33 appropriate referrals were accomplished. Several areas for improvement were identified and revised.

Conclusions: The M-PSC is a feasible and useful measure in enforcing follow-up care and promising to be an approach in standardizing post-stroke follow-up practice in telehealth during the coronavirus disease 2019 pandemic.

\section{Background}

With more than 10 million new cases annually, stroke has become a leading cause of mortality and disability worldwide[1]. The economic costs of long-term post-stroke care are substantial and the burden is expected to increase further as a result of the epidemiological transition from infections to noncommunicable diseases[2, 3]. However, there is no standardized process for long-term post-stroke care, though our health systems have been struggling to deliver effective interventions.

The global stroke community advisory panel developed an instrument named Post Stroke Checklist (PSC) to help health care professionals in standardizing the process of identifying long-term care after stroke and facilitating referrals[4]. It has been proved to be feasible and useful, furthermore, gained international recognition and endorsed by the World Stroke Organization to improve the follow-up care of post-stroke individuals. A Mandarin version of the PSC (M-PSC) was subsequently developed in keeping with guidelines[5]. Yet, whether the M-PSC or the original measure can only provide recommendations for each problem identified. Screenings and interventions are conducted or not depending on clinicians[6, 7]. It is not capable of enforcing follow-up care, and the direct referral might result in unnecessary waste of medical resources and increase the economic burden on individuals. We integrated the evidence summary into the M-PSC, added further screenings and interventions, and viewed it as a first step 
towards its application in the standardized post-stroke follow-up practice in telehealth during the coronavirus disease 2019 (COVID-19) pandemic. Besides, data regarding stroke severity and subtype are not discussed, which limits the feasibility and comparison of the PSC in different countries[6, 8]. We reported these issues and sought to explore the feasibility and usability of the M-PSC in a telephonebased follow-up. The objective of this study is to describe an evidence-based optimization of the M-PSC and evaluate its feasibility and usability in clinical practice.

\section{Methods}

\section{Aims}

The objective of this study is to describe an evidence-based optimization of the M-PSC and evaluate its feasibility and usability in clinical practice.

\section{Design}

A mixed methods study design was adopted to test the feasibility and usability of the M-PSC in a telephone-based follow-up. The M-PSC was not used for a performance evaluation, so psychometric appraisals, such as interrater reliability and criterion and construct validity, were not required[9]. The qualitative method was applied in the focus group discussion and the debriefing meeting in consonance with criteria for qualitative studies [10]. To provide thorough reporting of the study both STROBE and COREQ statements were used (Additional file 1).

\section{Instrument}

The M-PSC is an 11-item checklist administered by health care professionals to poststroke individuals at 6 and 12 months and annually thereafter, which focuses on areas where interventions have the largest impact on stroke survivors' quality of life. There is a dichotomous 'yes'/ 'no' response scale without scoring of each item and a referral recommendation for each problem identified. The content validity index for each item of the M-PSC was from 0.83 to 1.00 while the average was 0.98 with a scale-content validity index greater than 0.90 . The 11 th item (Relationship with family) is recommended asking only if clinicians were close to stroke survivors' families and confident that action could be taken to improve their relationship. In the present study, we didn't ask this question. Further screenings and specific suggestions were given to the item of 'Secondary Prevention', 'Communication', 'Mood' and 'Cognition' based on studies of evidence summaries. For detailed instructions, refer to the Additional file 2.

\section{Participants}

Participants were consecutively recruited from six neurology departments and seven neurosurgery departments in a specialized hospital when discharged in November 2020. Stroke diagnosis was confirmed by brain computed tomography or brain magnetic resonance imaging. Inclusion criteria were adult participants, with good competence in Mandarin and a clinical diagnosis of stroke between October 2020 to November 2020. Stroke survivors undergoing end of life were excluded. Individuals with aphasia, 
cognitive impairment and impaired hearing were included if a proxy family member had consented to assist in the telephonic follow-up and signed the informed written consent. The smallest sample size required for testing the validity of an instrument ranges from 5 to 10 times the number of its items[11]. As item 11 was not asked, the estimated sample size was 50-100 stroke survivors. Considering the absence of the sample, we increased the sample size by $40 \%$, we should invite $84-167$ poststroke individuals to participate in this study. Clinicians were engaged across various professions.

\section{Data Collection}

Data were collected from April 2021 to May 2021. The clinicians enrolled in this study received specific training from a researcher including the needed instruction for a correct administration of the M-PSC and a simulation. A stroke survivor was present in the simulation and personal perceptions were discussed and audio-recorded. An observer took notes and ask questions to clarify issues raised if necessary. The trained clinicians contacted participants by telephone calls at six months since the most recent diagnosis. Participants who didn't respond were called a second time in one week. If no response was received, they were viewed as not interested in this study and not contacted further. Due to the limitation of the telephone call, screenings of the 'Communication', 'Mood' and 'Cognition' were conducted only if participants had consented to have a video chat via WeChat. A satisfaction questionnaire would be sent to the participant after the call while clinicians completed a satisfaction questionnaire and the Pragmatic Face and Content Validity Test (PRAC-Test) at the end of calls[12]. The time used and problems that occurred in telephone calls were also collected. Then, a debriefing meeting was held with clinicians and two researchers. The meeting was recorded and transcribed. Data on clinical characteristics were collected from medical records. Stroke severity was assessed with the National Institutes of Health Stroke Scale[13], intracerebral haemorrhage (ICH) score[14] and Hunt and Hess grade[15]. Ischemic strokes were classified according to the Trial of Org 10172 in Acute Stroke Treatment classification[16] whilst intracerebral haemorrhage subtypes were categorized as structural vascular lesions, medication, amyloid angiopathy, systemic disease, hypertension or undetermined[17], with the spontaneous subarachnoid haemorrhage (sSAH) as aneurysms, non-aneurysmal perimesencephalic haemorrhage or other causes[18].

\section{Data Analysis}

Quantitative data collected from stroke survivors, the M-PSC, satisfaction questionnaires and the PRACTest were analysed using descriptive statistics and SPSS 20.0 statistical software. Key themes of the focus group discussion and the debriefing meeting were identified and summarised, using thematic analysis techniques.

\section{Ethical Consideration}

This study was approved by the Ethics Committee of Nanfang Hospital (No. NFEC-2018-054). Participants were fully informed about the aim and procedure before the start of this study and gave 
written informed consent. If the participant was unable to give consent, the written informed consent could be obtained from a proxy family member.

\section{Results}

\section{Sample Characteristics}

A total of 167 individuals consented to take part in the study. 101 participants were assessed at six months and 12 participants were successfully contacted a second time. Three of them were excluded due to missing data, resulting in a study sample of 110 participants (Fig. 1). The average age of participants was $56.2 \pm 11.4$ years [range 19-85 years] with a large proportion of male participants $(70.9 \%)$ and married participants $(96.4 \%)$. The majority of participants were diagnosed with ischemic stroke (48.2\%) and ICH (36.4\%), 11 (10\%) participants suffered from SSAH and a diagnosis of mixed stroke was given to six participants (5.5\%). The characteristics are shown in Table 1.

Nine clinicians were enrolled in this study, including two resident doctors in neurology, one resident doctor in neurosurgery, two rehabilitation physicians, three clinical nurses and one stroke specialist nurse. Age ranged from 29 to 35 years old, and $66.7 \%$ of them were women. The average work years of stroke were 6.0 years [range $2-10$ years].

TABLE 1 Characteristics of participants 
Characteristics

Mean age, years (SD)

Male sex, n (\%)

Marital status, $\mathbf{n}(\%)$

Married

Single

Stroke subtype, n (\%)

Ischemic stroke

$\mathrm{ICH}$

SSAH

Mixed stroke

TOAST classification, $\mathbf{n}(\%)$

Large vessel disease

Cardioembolic disease

Small vessel disease

Other aetiology

Undetermined aetiology

NIHSS, $\mathbf{n}(\%)$

0-3

4-14

$15-20$

21-42

SMASH-U classification, $\mathbf{n}$ (\%)

Structural vascular lesions

Medication

Amyloid angiopathy

Hypertension

Undetermined

ICH score, $\mathbf{n}(\%)$

0

1

2

3

sSAH subtypes, $\mathbf{n}(\%)$

Aneurysms

Non-aneurysmal perimesencephalic haemorrhage

Hunt and Hess grade, n (\%)

II

III

IV

$\mathrm{V}$

Risk factors, $\mathbf{n}(\%)$

Hypertension

Diabetes mellitus
$\mathrm{N}=110$

$56.2 \quad(11.4)$

$78 \quad(70.9)$

$106 \quad$ (96.4)

4 (3.6)

$\mathrm{N}=110$

$53 \quad$ (48.2)

$40 \quad$ (36.4)

$11 \quad(10.0)$

$6 \quad$ (5.4)

$\mathrm{N}=53$

$39 \quad$ (73.6)

$3 \quad(5.7)$

$6 \quad$ (11.3)

$1 \quad(1.9)$

$4 \quad(7.5)$

$\mathrm{N}=53$

$20 \quad$ (37.7)

$31 \quad$ (58.5)

$1 \quad$ (1.9)

1 (1.9)

$\mathrm{N}=40$

$10 \quad(25.0)$

$2 \quad(5.0)$

3 (7.5)

$22 \quad$ (55.0)

$3 \quad$ (7.5)

$\mathrm{N}=40$

11 (27.5)

$12 \quad(30.0)$

$8 \quad(20.0)$

$9 \quad(22.5)$

$\mathrm{N}=11$

$9 \quad$ (81.8)

$2 \quad(18.2)$

$\mathrm{N}=11$

7 (63.6)

$1 \quad(9.1)$

$1 \quad(9.1)$

$2 \quad(18.2)$

$\mathrm{N}=110$

$73 \quad$ (66.4)

$25 \quad(22.7)$

(Continues)

\begin{tabular}{lll} 
& 7 & $(63.6)$ \\
& 1 & $(9.1)$ \\
& 1 & $(9.1)$ \\
$\mathrm{N}=110$ & 2 & $(18.2)$ \\
& 73 & $(66.4)$ \\
& 25 & $(22.7)$ \\
\hline
\end{tabular}

TABLE 1 (Continued)

Risk factors, n (\%)

Dyslipidaemia

Current smoking

Current alcohol consumption

Cardiac disease

Arterial disease

$\mathrm{N}=110$

Previous stroke

Chronic kidney disease

Current poststroke treatment, n (\%)

$\begin{array}{lll}\mathrm{N}=110 & & \\ & 25 & (22.7) \\ 10 & (9.1) \\ 2 & (1.8) \\ 12 & (10.9) \\ & 74 & (67.3) \\ & 9 & (8.2) \\ & 5 & (4.5) \\ \mathrm{N}=105 * & & \end{array}$


Antiplatelet

52

Anticholesterol

67

Anticoagulant

6

Antihypertensive

72

Antihyperglycemic

22

Rehabilitation therapy

35

Speech and language therapy

11

Antidepressant/ Antianxiety agents

Neuroprotective agents

6

Traditional Chinese medicine

33

Nootropics

Others

26

8

20

(49.5)

(63.8)

(5.7)

(68.6)

(21.0)

(33.3)

(10.5)

(5.7)

(31.4)

(24.8)

(7.6)

(19.0)

$S D$, standard deviation; ICH, intracerebral haemorrhage; sSAH, spontaneous subarachnoid haemorrhage; TOAST, Trial of Org 10172 in Acute Stroke Treatment; NIHSS, National Institutes of Health Stroke Scale; SMASH-U, structural vascular lesions (S), medication $(M)$, amyloid angiopathy $(A)$, systemic disease $(S)$, hypertension or $(H)$ undetermined $(U)$. * 5 participants discontinued medication without permission.

\section{Amendments after the Focus Group Discussion}

Three themes were generated in the 57 mins discussion: contents and the media, barriers, and orders of items. The M-PSC was considered valuable by all clinicians, especially the stroke specialist nurse and the stroke survivor. However, they also pointed out its insufficiencies in contents and the media. "It's really necessary to standardize the poststroke follow-up, and I think it is useful to make up this flaw. Ur ...But... The questions, like the dysphagia, skin integrity and the burden of caregivers were also needed." "The suggestions on secondary prevention need to be more detailed" (Clinicians). "If we can communicate face to face or by video calls, it will be better. Thus, you can see how my fingers are moving" (The stroke survivor). Others viewed the detailed contents, the time used to administrate the M-PSC and the limitation of medical resources in rural areas as barriers. "As you see, we used nearly six minutes in the simulation. In that way, we must spend more time on the assessment. There is no need to add new items." "That is not easy to get such a 'recommendation' in rural areas, you know" (Clinicians). The order of the item was also an argument. "It will be better to ask questions according to their status at discharge. The orders...it is very important." "If many irrelevant questions were asked at first, they would suspect that you don't understand the conditions of their disease" (Clinicians).

Two open-ended questions were added and clinicians were permitted to conduct the assessment based on the feature of individual conditions and status at discharge. The topic of "Mixing the item of Activity of Daily Living (ADL) and Mobility" was found to be a dilemma and shelved for a further discussion after the follow-up.

\section{Feasibility of the M-PSC}

The M-PSC identified a wide range of unmet needs related to stroke (Fig.2). The most frequently reported problem for participants was life after stroke, reported by $40.0 \%$ of participants. Mobility was also frequently reported (39.1\%), as was ADL (35.5\%). Lack of secondary prevention was the least frequently 
reported problem, reported by $7.3 \%$ of participants, while the absence of life style changes and medication suggestions (subitems of Secondary Prevention) was higher, reported by $24.5 \%$ and $7.3 \%$ of participants. Meanwhile, the prevalence of mood changes (18.2\%) was higher than its subitems $(9.1 \%$ and $14.5 \%) .33$ participants (76.7\%) with mobility difficulties were continuing to receive rehabilitation therapy. In the $76.4 \%$ of participants, at least one need was identified. $81.0 \%$ of them indicated that all their problems were included in closed questions of the M-PSC and didn't mention other domains of possible issues. 13 participants consulted other stroke-related questions, such as dizziness, eyesight problems and ear problems, whist four participants reported other questions, including chest distress and coronavirus vaccine. The M-PSC was not applicable if individuals were in a coma, stupor and hemiplegia with aphasia, reported by four participants. The average time taken to administer the M-PSC was 8 mins [range 3'07"-27'39"].

\section{The Content and Face Validity}

Clinician satisfaction of the M-PSC was high, with an average rating of 9.6/10 in overall use and 9.0/10 in helping identify the needs. Data of the referral were not promising with an average scoring of 8.3/10. Participant satisfaction with the assessment was higher than 8.5 with 9.7/10 in identifying needs and $8.5 / 10$ in the overall assessment. In terms of the overall view of the M-PSC, clinician feedback was positive. The average rating measured by PRAC-Test was higher than 9.0 except for the item of 'exhaustive' (8.9/10) and 'quick to complete' (8.6/10).

\section{Further Screenings and Referrals}

Only one participant refused the further assessment. Totally 31 screenings were conducted implicating 20 participants, and 12 of them (60\%) were confirmed needing further treatments, culminating in six referrals to the psychologist, three referrals to the specialist speech and language therapist and two referrals to the neurosurgeon. Four of them were also transferred to the rehabilitation physician considering post-stroke sequelae. A stroke survivor didn't get the help due to the limitation in rural areas and the personal financial status.

Five participants were suggested to go to a diabetes clinic and one of them was also advised to the cardiac clinic for chest pain. Eight participants described the absence of secondary prevention, then risk factor assessments and treatments were carried out by staff in primary health-care institutions. Four participants were referred to the neurologist owing to the spasticity or pain after stroke. One participant didn't get such a medical resource due to the same reason. In the end, 33 appropriate treatment referrals were accomplished.

\section{Amendments after the Debriefing Meeting}

The debriefing meeting lasted 34 mins. Problems that occurred in the assessment were discussed and several areas for improvement were identified, covering: (1) suggestions on secondary prevention must be told to stroke survivors whether they had received related advice before, (2) the entries of the Barthel 
Index substituted questions of the ADL and mobility, and became a new item. The overlap in subitems and the same suggestion were found while family members reported they would not let the stroke survivors do such a dangerous thing, like boiling water, pouring boiled water, preparing meals and going outside alone. In addition, clinicians agreed that feeding was an important thing for stroke survivors in China, (3) minor revisions on referral recommendations (Additional file 2).

\section{Discussion}

This study indicates that the M-PSC is a feasible and useful measure in identifying long-term post-stroke needs and enforcing follow-up care, and expected to be an approach in standardizing post-stroke followup practice in telehealth during the COVID-19 pandemic.

The feedback from participants and clinicians implied a high satisfaction with the overall use of the MPSC and its ability in identifying needs after stroke, furthermore, clinician feedback on the content and use of the M-PSC was generally positive, thus demonstrating great face and content validity. Clinicians were slightly confident that the M-PSC was exhaustive and quick to complete, which was also reported in other studies. Yet, we didn't view this as a big problem. A sound checklist highlights the essential criteria that should be considered in a particular area[19]. The findings indicated that the M-PSC was able to identify a wide range of unmet needs in poststroke survivors. Indeed, all problems reported by participants were involved in the M-PSC with 10 closed questions and two open-ended questions. The results also demonstrated that the item decomposition of the PSC was necessary, which was reported in our previous research (not published). The ratio of the item and subitem was not matching and there was even a triple deviation with them. $40 \%$ of participants who reported difficulties with communication, cognition, or mood were verified not needing further treatments. With additional screenings and interventions following evidence summaries, health resources were saved, in the meanwhile, the time cost to conduct the assessment was not prolonged: Italy (73\%ष5 mins)[7], UK (mean 13 mins)[6], Singapore (mean 17 mins)[6], Sweden (median time 30 mins)[20]. We present a comprehensive picture of patient characteristics. The study population was consecutively enrolled from 13 departments including individuals with aphasia, cognition impairment and impaired hearing. Though the proportion was not matching the national cohort due to the simultaneous recruitment in neurology and neurosurgery departments, we thought it turned out to be a benefit to exploring the feasibility and usability of the MPSC in ICH and SSAH, which was not reported before. The M-PSC was found to be unsuitable for individuals with coma, stupor and hemiplegia with aphasia, reported by three participants with ICH and one participant with ischemic stroke. For others with ICH or SSAH, the evaluation of the M-PSC was appreciated. In this study, ongoing functional difficulties were common at six months post-stroke and affected $76.4 \%$ of participants. The ratio was lower than other studies[8, 20, 21], which was potentially caused by the differences across the sample. The top three prevalence of needs were life after stroke, mobility and ADL as with John's study, whilst the proportion of participants who received rehabilitation therapy were higher than his, though the majority of participants in both studies received the education on preventing further strokes[8]. This could be due to the economic disparity in China. Comparing worldwide, specific unmet needs were more frequently reported in the domains of life after stroke, mood, 
cognition, mobility and ADL[6-8, 20,21]. However, we believe compassion may be more reasonable in a similar baseline.

We strongly recommend using the 11-item M-PSC if clinicians are close to the family of stroke survivors and inviting the family to participate in the follow-up since individuals with stroke might be ashamed to describe difficulty in the body parts directly. Yet, how to help them overcoming stigma, especially those with disabilities, is still a matter of discussion. Two objective realities also need to be considered. One is the insufficient resources in rural areas. In this study, two participants didn't receive rehabilitation therapy seeing the limitation of medical resources and personal financial status. The free home rehabilitation education based on evidence might be effective[22]. We have developed a WeChat mini-program named "Brain-Friendly Health Assistant". In the next step, we will add new modules of home rehabilitation according to the current best evidence and try to use it as the media to administrate the M-PSC in telehealth during the COVID-19 pandemic. The second problem ensues. Which is better for individuals to use the M-PSC, self-administered or interviewer-administered? Pre-completing the M-PSC can reduce appointment length and increase individual satisfaction[23]. However, we must acknowledge that there is discordance between the clinician and the individual interpretation of certain questions. The best approach might be a mixed management model.

\section{Limitations}

This study has several limitations. Firstly, the information regarding post-stroke problems was selfreported so memory errors are expected and expectation bias may have influenced the assessment. Secondly, although the sample size met the requirement of the instrument, the feasibility of results was still limited since only participants admitted to one specialized hospital were enrolled. Further research is needed to test the generalizability of the M-PSC. Finally, some participants were lost to follow up at six months.

\section{Conclusion}

This study has demonstrated that the M-PSC is a feasible and useful measure not only in identifying long-term outcomes but also in enforcing follow-up care for stroke. Further studies should consider the participation of the family and test the generalizability of the M-PSC while the insufficient resources in rural areas and the mode of administering the M-PSC require further discussion.

\section{Abbreviations}

PSC: Post Stroke Checklist

M-PSC: Mandarin version of the PSC

COVID-19: Coronavirus Disease 2019 
PRAC-Test: Pragmatic Face and Content Validity Test

ICH: Intracerebral Haemorrhage

sSAH: Spontaneous Subarachnoid Haemorrhage

ADL: Activity of Daily Living

\section{Declarations}

\section{Ethics Approval and Consent to Participate}

This study was approved by the Ethics Committee of Nanfang Hospital (No. NFEC-2018-054), all methods were carried out in accordance with relevant guidelines and regulations. Participants were fully informed about the aim and procedure before the start of this study and gave written informed consent. If the participant was unable to give consent, the written informed consent could be obtained from a proxy family member.

\section{Consent for Publication}

Not applicable, all data is anonymised.

\section{Availability of Data and Materials}

The datasets generated and/or analysed during the current study are not publicly available due to the need to maintain the anonymity of participants but are available from the corresponding author on reasonable request.

\section{Competing Interests}

The authors declare that they have no competing interests.

\section{Funding}

This work was supported by the grant from the Evidence-Based Nursing program of Nanfang Hospital, Guangdong, China (2021EBNa002) in the decision to submit the article for publication.

\section{Authors' contributions}

All authors have made substantial contributions to the conception and design, and interpretation of data. $\mathrm{YL}$ and $\mathrm{ZHL}$ were involved in recruitment and data verification. MJ was present as a facilitator in the focus group discussion and the debriefing meeting while HQL was an observer. SMH and JJL transcribed interviews, with JYT checking transcripts. Data analysis and interpretation were led by JYT and YL, with $\mathrm{HQL}$ contributing. MJ wrote the manuscript and all other authors critically revised the manuscript for important intellectual content. All authors read and approved the final manuscript and agree with 
accountability for all aspects of the work in ensuring investigating and resolving questions related to the accuracy or integrity of any part of the work.

\section{Acknowledgements}

The authors would like to express their gratitude to Mapi Research Trust for approval to use the PRACTest, to clinicians and participants who took part in this study, and to Nanfang hospital for supporting this study.

\section{References}

1. GBD 2016 Stroke Collaborators: Global, regional, and national burden of stroke, 1990-2016: a systematic analysis for the Global Burden of Disease Study 2016. LANCET NEUROL 2019, 18(5):439-458.

2. Rochmah TN, Rahmawati IT, Dahlui M, Budiarto W, Bilqis N: Economic Burden of Stroke Disease: A Systematic Review. Int J Environ Res Public Health 2021, 18(14):7552.

3. Zhu D, Shi X, Nicholas S, Chen S, Ding R, Huang L, Ma Y, He P: Medical Service Utilization and Direct Medical Cost of Stroke in Urban China. Int J Health Policy Manag. 2020. DOI: 10.34172/ijhpm.2020.111.

4. Philp I, Brainin M, Walker MF, Ward AB, Gillard P, Shields AL, Norrving B: Development of a Poststroke Checklist to Standardize Follow-up Care for Stroke Survivors. Journal of Stroke and Cerebrovascular Diseases 2013, 22(7): e173-e180.

5. Wild D, Grove A, Martin M, Eremenco S, McElroy S, Verjee-Lorenz A, Erikson P: Principles of Good Practice for the Translation and Cultural Adaptation Process for Patient-Reported Outcomes (PRO) Measures: report of the ISPOR Task Force for Translation and Cultural Adaptation. VALUE HEALTH 2005, 8(2):94-104.

6. Ward AB, Chen C, Norrving B, Gillard P, Walker MF, Blackburn S, Holloway L, Brainin M, Philp I: Evaluation of the Post Stroke Checklist: A Pilot Study in the United Kingdom and Singapore. INT J STROKE 2014, 9(SA100):76-84.

7. Iosa M, Lupo A, Morone G, Baricich A, Picelli A, Panza G, Smania N, Cisari C, Sandrini G, Paolucci S: Post Soft Care: Italian implementation of a post-stroke checklist software for primary care and identification of unmet needs in community-dwelling patients. NEUROL SCI 2018, 39(1):135-139.

8. Olver J, Yang S, Fedele B, Ni J, Frayne J, Shen G, McKenzie D: Post Stroke Outcome: Global Insight into Persisting Sequelae Using the Post Stroke Checklist. Journal of Stroke and Cerebrovascular Diseases 2021, 30(4):105612.

9. Burian BK, Clebone A, Dismukes K, Ruskin KJ: More Than a Tick Box: Medical Checklist Development, Design, and Use. ANESTH ANALG 2018, 126(1):223-232. 
10. Tong A, Sainsbury P, Craig J: Consolidated criteria for reporting qualitative research (COREQ): a 32item checklist for interviews and focus groups. Int J Qual Health Care 2007, 19(6):349-357.

11. Sun Z, Xu Y: Medical Statistics. 4rd ed. Beijing: People's Medical Publishing House; 2014.

12. Bagneux V, Barnes N, Arnold B: Development of a standardized face and content validity test to evaluate patient questionnaires for clinical practice. PRO Newsletter 2007, 39:12-14.

13. Brott T, Adams HJ, Olinger CP, Marler JR, Barsan WG, Biller J, Spilker J, Holleran R, Eberle R, Hertzberg $\checkmark$ et al: Measurements of acute cerebral infarction: a clinical examination scale. STROKE 1989, 20(7):864-870.

14. Hemphill JR, Bonovich DC, Besmertis L, Manley GT, Johnston SC: The ICH score: a simple, reliable grading scale for intracerebral hemorrhage. STROKE 2001, 32(4):891-897.

15. Hunt WE, Hess RM: Surgical risk as related to time of intervention in the repair of intracranial aneurysms. J NEUROSURG 1968, 28(1):14-20.

16. Adams HJ, Bendixen BH, Kappelle LJ, Biller J, Love BB, Gordon DL, Marsh ER: Classification of subtype of acute ischemic stroke. Definitions for use in a multicenter clinical trial. TOAST. Trial of Org 10172 in Acute Stroke Treatment. STROKE 1993, 24(1):35-41.

17. Meretoja A, Strbian D, Putaala J, Curtze S, Haapaniemi E, Mustanoja S, Sairanen T, Satopaa J, Silvennoinen H, Niemela M et al: SMASH-U: a proposal for etiologic classification of intracerebral hemorrhage. STROKE 2012, 43(10):2592-2597.

18. Macdonald RL, Schweizer TA: Spontaneous subarachnoid haemorrhage. LANCET 2017, 389(10069):655-666.

19. Hales BM, Pronovost PJ: The checklist-a tool for error management and performance improvement. J CRIT CARE 2006, 21(3):231-235.

20. Ullberg T, Månsson K, Berhin I, Pessah-Rasmussen H: Comprehensive and Structured 3-month Stroke Follow-up Using the Post-stroke Checklist (The Struct-FU study): A Feasibility and Explorative Study. Journal of Stroke and Cerebrovascular Diseases 2021, 30(2):105482.

21. Hotter B, Padberg I, Liebenau A, Knispel P, Heel S, Steube D, Wissel J, Wellwood I, Meisel A: Identifying unmet needs in long-term stroke care using in-depth assessment and the Post-Stroke Checklist - The Managing Aftercare for Stroke (MAS-I) study. Eur Stroke J 2018, 3(3):237-245.

22. Chi NF, Huang YC, Chiu HY, Chang HJ, Huang HC: Systematic Review and Meta-Analysis of HomeBased Rehabilitation on Improving Physical Function Among Home-Dwelling Patients With a Stroke. Arch Phys Med Rehabil 2020, 101 (2):359-373. 
23. Turner GM, Mullis R, Lim L, Kreit L, Mant J: Using a checklist to facilitate management of long-term care needs after stroke: insights from focus groups and a feasibility study. BMC FAM PRACT 2019, 20(1):2.

\section{Figures}

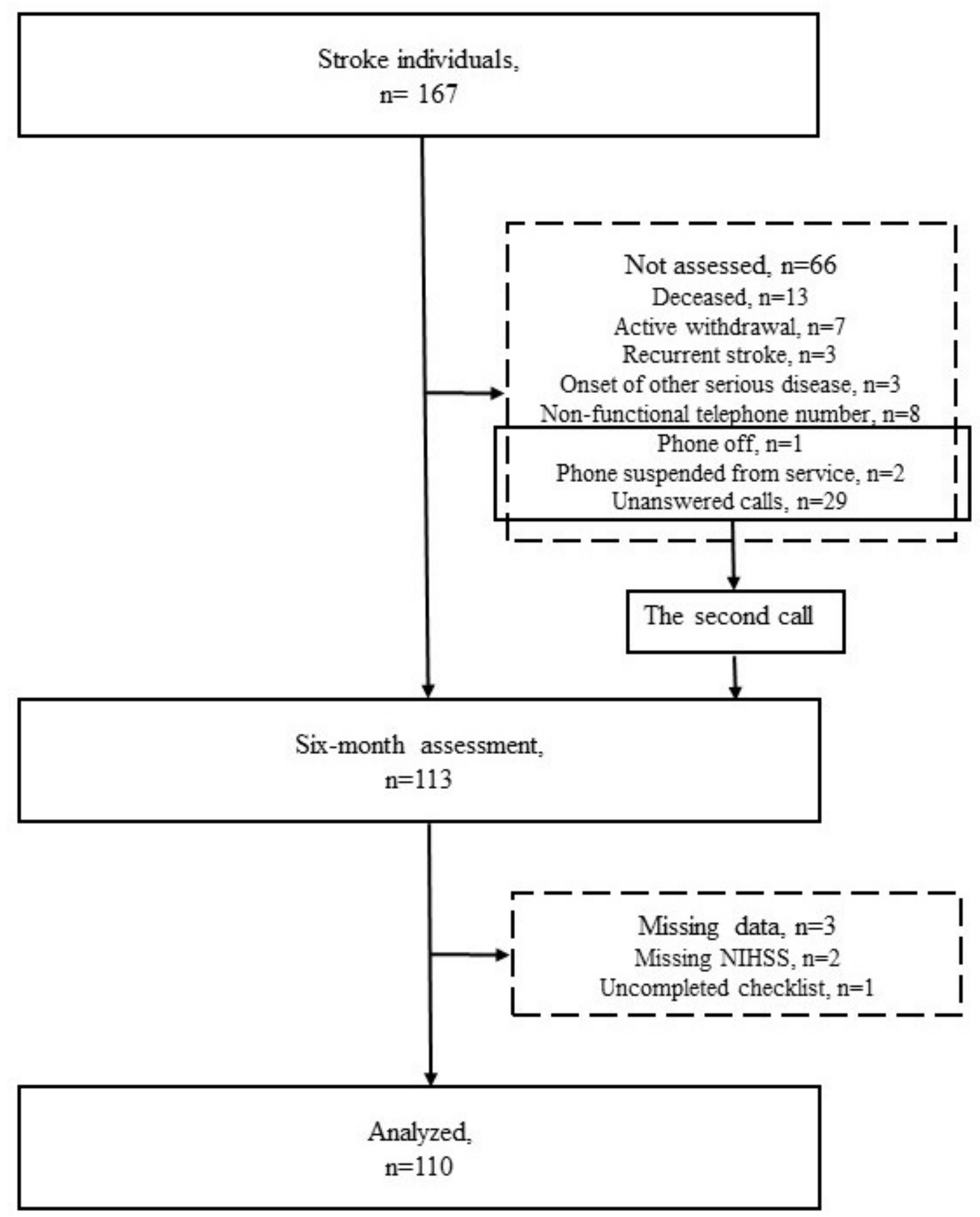


Flowchart of participants included in the study.

\section{The M-PSC item}

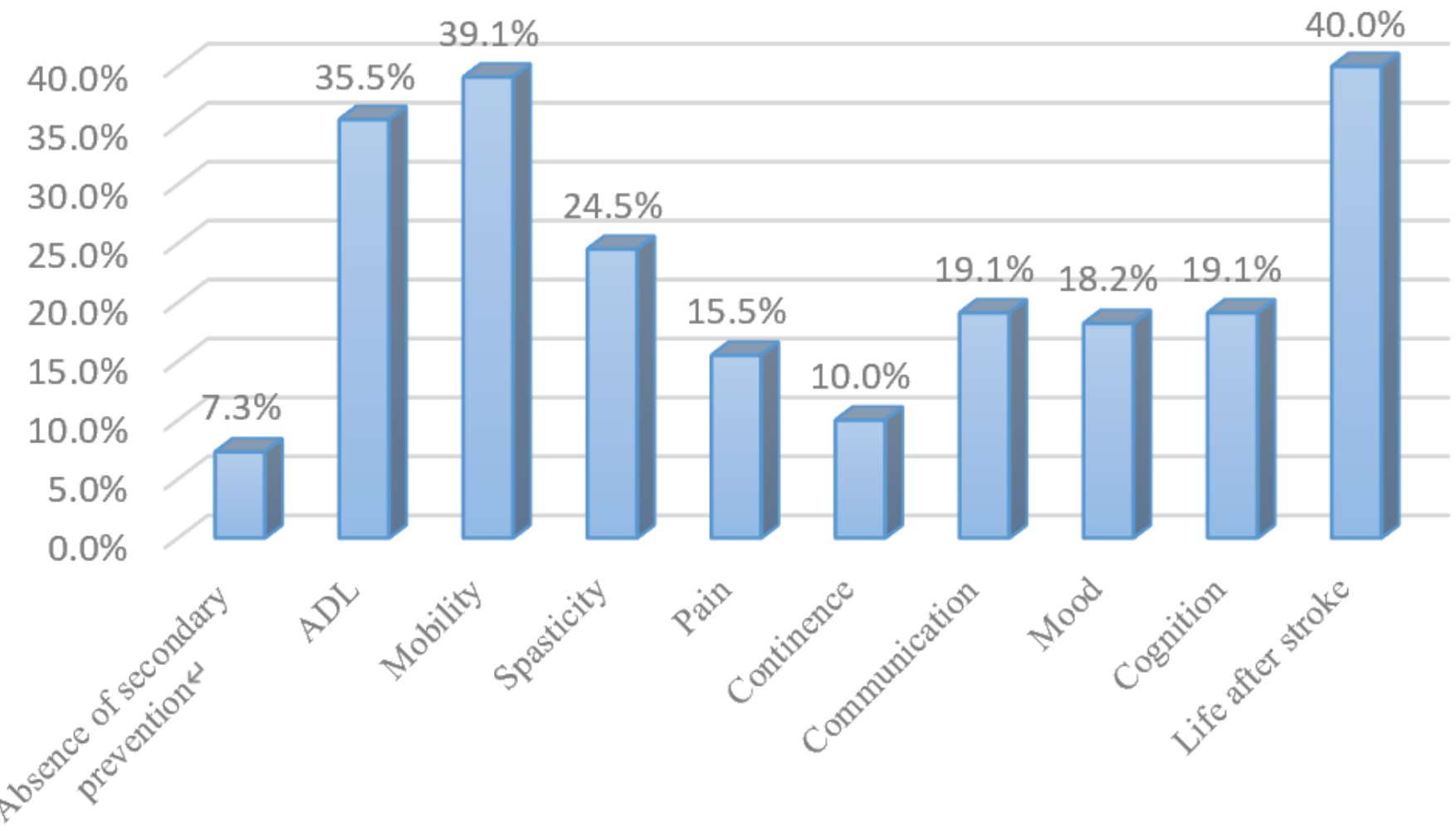

Figure 2

Unmet needs identified with the M-PSC. M-PSC, the Mandarin version of the Post Stroke Checklist; ADL, the Activity of Daily Living.

\section{Supplementary Files}

This is a list of supplementary files associated with this preprint. Click to download.

- STROBEandCOREQstatements.pdf

- SupportingInformation.pdf 